\section{IPALION: A FORTRAN IV program for comparing interpersonal perceptions in dyads}

\author{
THEODORE G. GROVE \\ Portland State University, Portland, Oregon 97207 \\ and

\section{JAMES HAYS} \\ Columbia Regional Association of Governments, \\ Portland, Oregon 97207
}

Comparisons of interpersonal perceptions as a technique of inquiry in psychotherapy, education, marriage counseling, and business management, and in other communication-intensive contexts, have evoked widespread interest by workers in a variety of disciplines (e.g., Bryant, 1974; Drewery, 1969; Goffman, 1959; Newcomb, 1961; Taguiri, 1958; Watzlawick, Beavin, \& Jackson, 1967). Many of these investigators have maintained that, since the meaning of behavior to interacting persons is mediated through the interplay of perceptions each has of the other, data on interpersonal perception are essential to a fuller understanding of dyadic behavior systems. Acquisition of such data entails somehow eliciting information about the interperceptions of members of a dyad across different cognitive and affective realms of their relationship and subsequently comparing selected perceptions of the two parties in order to isolate areas of accuracy and inaccuracy in their perceptual relationship. ${ }^{1}$

One of the most widely known efforts in this regard is the interpersonal perception method (IPM) developed by Laing, Phillipson, and Lee (1966), which represents an extension of Dymond's (1949) early work on interpersonal perception. Laing et al. report a method and an instrument for examining the interperceptions of husband-wife dyads that entails comparing pairs of perceptions with respect to specified issues in their relationship (e.g., "respects," "takes seriously," "hates," etc.). Such comparisons at several perceptual levels provide a glimpse of how accurately members of a pair can identify each other's perceptions. Recently, Alperson (1975) has demonstrated the logical integrity of the IPM and the inferences derived from it concerning "agreement," "understanding," and "realization."

Despite the promise and logical versatility of this method, a number of investigators suggest why so little refinement or application of the IPM has been attempted. Alperson (1975) indicates that the "scoring procedures are tedious and difficult." Kotkas (1969) writes that the original IPM questionnaire "entails a

This work was supported in part by Research and Publications Award 90-050-5301-GAB from Portland State University. time consuming scoring process." The IPM and the associated questionnaire require 720 responses from each partner-12 responses to each of 60 issues. Once obtained, additional hours are required to code, score, and interpret a single episode in a dyadic relationship. Presumably because of the difficulties noted above, the writers are unable to find any validity or reliability studies, after more than a decade since the original report.

At present, only a single effort toward developing a program for computerized data processing has been reported. Although laudable, this rudimentary version does not retain the comprehensiveness and detail of the original IPM required for a complete analysis of matched perceptions. Neither does it compute summary scores for either partner or for the dyad as a whole. In addition, its reported form does not provide the information investigators in the field need in order to determine its usefulness for their data collection efforts.

The program described in this report provides more detailed information than the original IPM hand-scoring method, as well as 84 summary scores per dyad. Additional options add to its flexibility in supporting either a research or a clinical-diagnostic data collection goal. These include options for processing a variable number of dyads at one time (up to 100 ) and a variable number of issues for each dyad (up to 100).

The Program. IPALION (interpersonal perception) is a FORTRAN IV program for scoring an instrument based on the logic of the IPM. This program improves on the IPM model in several ways in addition to those already cited. Some indices appearing in the original data summaries (Laing et al., 1966, pp. 75-80) may be directly inferred from other indices and are therefore redundant. For example, "disagree" totals are complementary to their "agree" counterparts. These and other redundant indices have been removed in the present program, thereby simplifying the data summaries for each dyad. Also, the language and arrangement of questions on the original questionnaire have been modified to (1) provide a clearer question form, and (2) obviate the need for female respondents to transform pronouns prior to their responses.

The two parties to the dyad are designated "P" (person) and " $O$ " (other). The instrument is divided into some given number of issues, each referring to a particular relational attribute (e.g., respects, loves, takes advantage of). Each issue is further subdivided into four aspects and three levels of perception. The four aspects of an issue consist of the four combinations of person and other (i.e., PO, OP, PP, and OO). For example, using the issue of "respect," these aspects become: I respect him, he respects me, I respect myself, and, he respects himself.

The three levels or perspectives are the direct, the 
understanding, and the realization levels. At the direct level, each member affirms or denies each of the four aspects of an issue: I respect him, and so on. At the understanding level, each predicts how the partner will respond to the same set of questions. At the realization level, each registers the predictions he thinks the partner will make regarding his own direct responses. Thus, each member of the dyad responds to 12 items (4 aspects $\times 3$ levels) for every issue, each response requiring a binary decision (yes or no). ${ }^{3}$

The five-letter clusters in the profiles section display the five possible outcomes of comparing the six responses, three each from $\mathrm{P}$ and $\mathrm{O}$, relating to a particular aspect-issue combination. Matched perceptions at the direct level are termed agreements and coded as "A," while mismatched perceptions are recorded as "D." For example, if P responds "yes" to "I respect her," while $O$ responds "yes" to "he respects me," this constitutes agreement for the $P O$ aspect. If $P$ responds "yes" to "I respect myself," while O responds "no" to "he respects himself," that issue under aspect PP would be scored "D."

The second level of comparison is called understanding or misunderstanding and is coded $U$ or $M$, respectively. This is determined by comparing P's direct response with $O$ 's understanding response, and vice versa. For example, if $P$ correctly predicts (understands) O's response of "no," this would constitute a match (U). If $O$ predicts that $P$ would respond "no" to "he respects me," then $O$ misunderstands (M) P's perception, and an $\mathrm{M}$ is coded. By convention, P's perceptions are coded to the left of middle and O's to the right in the five-letter clusters.

The third level of comparison is realization or failure to realize and is coded $R$ or $F$, respectively. The outcome is determined by comparing each party's realization response with the other's understanding response. For example, if $\mathrm{P}$ felt that $\mathrm{O}$ understood his (P's) direct response, but $\mathrm{O}$ actually misunderstood, then $P$ failed to realize that $O$ misunderstood and receives an $F$. In the other direction, if $O$ felt that $P$ misunderstood him and $P$ did, then $O$ realizes that $P$ misunderstands and receives an $\mathrm{R}$.

The logic of each of the five comparisons for each cluster is presented through an example cluster of FUDMR for the OP aspect in Figure 1. A perfect cluster of matches would be RUAUR; a perfect cluster of mismatches would be represented by FMDMF.

The second section of the output consists of summaries across all issues in the questionnaire of the various degrees of perceptual matching for $\mathrm{P}, \mathrm{O}$, and the D total. Also, several conditional matches (numbers 8-16), such as "understood agreements" are displayed. All results are reported as absolute scores and as percentages. The first seven (nonconditional) indices are further broken down into the interpersonal (PO + OP) and the self-concept $(\mathrm{PP}+\mathrm{OO})$ relational aspects. There are 16 indices in all. The last index compares each party's realization responses with his own direct responses, giving the extent to which each party feels

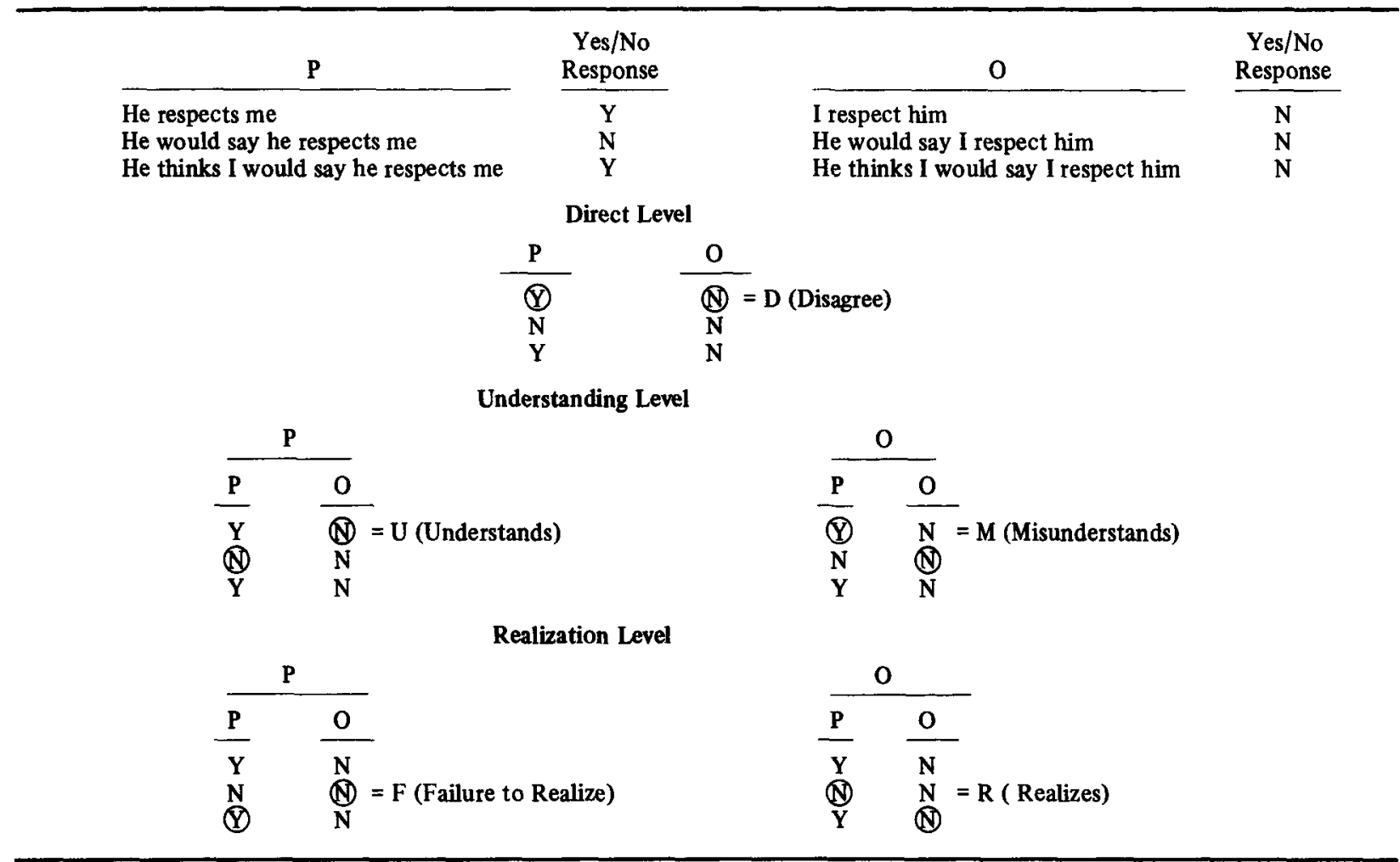


understood. Thus, P feels understood whenever he realizes that $\mathrm{O}$ understands him and also whenever he fails to realize that $\mathrm{O}$ misunderstands him.

Summary. IPALION is a computer program for comparing interpersonal perceptions between members of a dyad. This program provides full information on three levels of perception matching for any pair of individuals, permitting a variable number of dyads per batch and a variable number of relational issues per dyad. The printout provides more information than the original hand-scoring method, including 84 summary scores per dyad, a record of the outcome of every set of compared perceptions, and a variety of nonconditional and conditional indices.

In addition to total dyad summaries, the printout displays separate outcomes for both members of the pair and also separates agreement, understanding, and realization outcomes into interpersonal and self-concept aspects of the perceptual relationship. IPALION could provide investigators with a useful tool for examining areas of interpersonal relationships at a level of detail and to an extent that was formerly not feasible.

\section{REFERENCES}

Alperson, B. L. A Boolean analysis of interpersonal perception. Human Relations, 1975, 28, 627.652.
BRYANT, B. K. Locus of control related to teacher-child interperceptual experiences. Child Development, 1974, 157-164.

Cronbach, L. J. Processes affecting scores on "understanding of others" and "assumed similarity." Psychological Bulletin, $1955,52,177-193$.

Drewery, J. An interpersonal perception technique. British Journal of Medical Psychology, 1969, 42, 171-181.

Dymond, R. F. A scale for the measurement of empathic ability. Journal of Consulting Psychology, 1949, 13, 127-133.

Goffman, E. Strategic interaction. Philadelphia: University of Pennsylvania Press, 1959.

KotKas, L. J. Informal use of the "interpersonal perception method" in marital therapy. Canadian Psychiatric Association Journal, 1969, 14, 11-14.

Laing, R. D., Phillipson, H., \& Lee, A. R. Interpersonal perception. New York: Springer, 1966.

Newcomв, T. M. The acquaintance process. New York: Holt, Rinehart, \& Winston, 1961.

TAGUIRI, R. Social preference and its perception. In R. Taguiri \& L. Petrullo (Eds.), Person perception and interpersonal behavior. Stanford: Stanford University Press, 1958.

Watzlawick, P., Beavin, J. H., \& Jackson, D. D. Pragmatics of human communication. New York: Norton, 1967.

\section{NOTES}

1. For an excellent discussion of the problems of interpreting dyadic scores in research designs, see Cronbach (1955).

2. For a detailed discussion of these and other attributes of the IPM, see Laing et al. (1966, pp. 49-72).

3 . The authors modified the original multilevel response format.

(Accepted for publication July 6, 1978.) 